\title{
A Generalized Interaction Wake Model with Its Variation for Control in Wind Farms
}

\author{
Lin Pan ${ }^{1,2}$, Holger Voos ${ }^{1}$, Yongping Pan ${ }^{3}$, Mohamed Darouach ${ }^{4}$ \\ 1. Interdisciplinary Centre for Security, Reliability and Trust, University of Luxembourg, Luxembourg \\ E-mail: lin.pan@uni.lu, holger.voos@uni.lu \\ 2. School of Electric and Electronic Engineering, Wuhan Polytechnic University, Wuhan 430023, China \\ 3. School of Biomedical Engineering, National University of Singapore, Singapore 117575, Singapore \\ E-mail: biepany@ nus.edu.sg \\ 4. Research Center for Automatic Control of Nancy, University of Lorraine, France \\ E-mail: mohamed.darouach@univ-lorraine.fr
}

\begin{abstract}
In this paper, an Improved Frandsen Wake Model(IFWM) and its variation model-Improved Frandsen Normal Distribution Wake Model(IFNDWM) for Wind Farms(WFs) have been presented and analyzed exhaustively with mathematic forms. The comparisons of these two different wake models of WFs also have been expounded. Meanwhile, experimental comparisons of both Wake Models are studied. Further, focused on the WFs of Luxembourg, the statistical methods are used to model the distribution of Wind Speed(WS) and Wind Direction(WD). Finally, some simulations are provided to test and verify their effectiveness and correctness of these models. Wind Rose(WR) and wind resources demonstrate Wind Power (WP) in full. These assessments are expected to enhance WP exploitation and utilization in WFs of Luxembourg.
\end{abstract}

Key Words: Coordinated Control, Wind Farms(WFs), Wind Turbine(WT), Wind Rose(WR), Wind Power (WP), Tip Speed Ratio(TSR), Large-Eddy Simulation(LES)

\section{Introduction}

It is well known that wind is renewable, abundant and pollution-free, it is one of the fastest growing energy sources. With the increase of global wind-generation capacity in the last ten years, Wind Energy(WE) has become the fastestgrowing electrical energy in the world. Since WE is widely recognized to be one of the most important renewable energy sources, Wind Power(WP) assessment is a critical step in the processes of WE utilization. In order to enhance the effective utilization of WE, the best way is to utilize existing WFs through improving control techniques and algorithms. At present, WE systems are being inclined to develop into large-scale distributed and coordination systems where there are even more than eighty individual Wind Turbines(WTs) in operation. In contrast to the conventional power plants, e.g. nuclear power, thermal power, hydropower, etc. [1-7], these wind devices and equipment are expected to operate and provide high quality power (Such as: Safe, Stable, Controllable and Predictable (SSCP)) at the lowest possible cost.

Generally, WTs not only generate power but also induce wakes behind their swept areas in the WFs. For the downstream WTs, the wakes will weaken the WP generation performance. Due to the wake effect, the layout of a WFs greatly reduces the upper boundary of their potential WP generation. This study aims to design and optimal the WFs layout and even avoid the wake loss through the distribution of WTs. Meanwhile, because of the aerodynamic interactions among the array WTs, using control algorithms, optimizing WP capture of WFs with coordination and optimization control will no doubt increase the effective utilization of WE. One method for dealing with these aerodynamic interaction$\mathrm{s}$ is to develop and use wake models in the distributed and optimization control algorithms. An alternative method is to develop an online control approach where each WT ad-

This work is supported by AFR and FNR programs of Luxembourg. justs its own induction model coefficients according to the information of local WFs, such as the WP generated by individual WT, local wind conditions, local wind speed, local WD, local density of air, or interacted information regarding neighbor WTs [8, 9].

It is notable that WE develops rapidly in Luxembourg among recent years. In view of Luxembourg locating in the western central area of European, and there are abundant wind resources to tap into Luxembourg. There are currently more than 16 WFs established in the different places of Luxembourg. As an example, Fig. 1 shows WF Wandpark Burer Bierg/Enovos-Burer Bierg, which is one of the largest WFs in Luxembourg $[10,11]$.

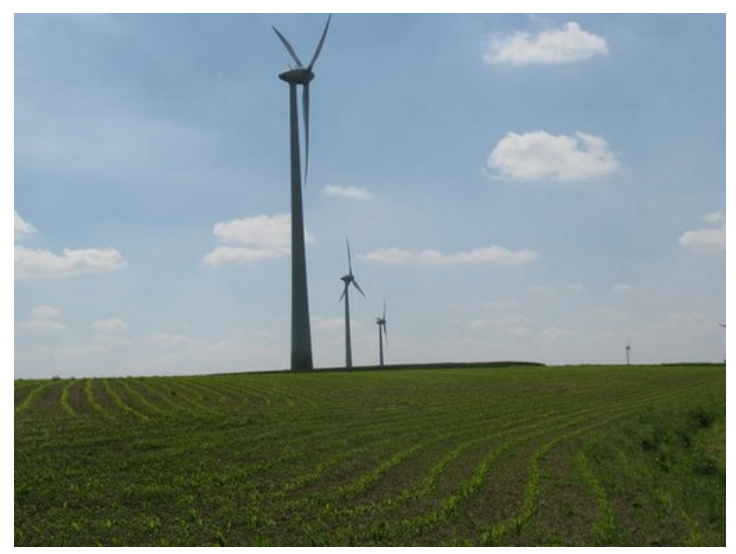

Fig. 1: The portraits of Wandpark Burer Bierg/Enovos-Burer Bierg WF in Luxembourg[11].

From control perspective, the WF level control is the most challenging task. The complexities of large scale WFs are handled by hierarchical or multi-level approaches, separating in the control of the following levels, for example, the WF level and control on the single WT level [12]. The opera- 
tor level controllers serve the demands from the network operator that give a set points for active and reactive power for the whole WF combined with several operational modes, e.g. maximum WP production, rate limiting, balancing, frequency control, voltages control, or delta control. On the lowest control level, the single WT level, the respective set points are achieved via coordinated control of a wind turbine array for power maximization which is described in [13]. Meanwhile, the most challenging task is the farm level control. Inspired by the above works and discussions, we argue that the key and core research contents are to operate WFs with the efficient methods, to improve WP, power quality, and energy capture of the overall WTs. Moreover, for reducing complex structural electricity loading, advanced and hybrid control measures have to be applied on the WFs. However, there is still lack of effective control method of WFs which requires further research and development.

The main contributions of this paper are as follows. Firstly, an Improved Frandsen Wake Model(IFWM) and its variation model-Improved Frandsen Normal Distribution Wake Model(IFNDWM) for WFs have been presented and analyzed with mathematic forms in detail. After that, comparisons of these two different wake models of WFs have been expounded. Further, experimental comparisons of both Wake Models is studied. Finally, focused on the WFs of Luxembourg, wind rose and wind weibull probability density distribution are elaborated and discussed. Some simulation figures are provided to test and verify their effectiveness and correctness in the paper.

The paper is organized as follows: In sections 2 and 3, the IFWM and IFNDWM are described and deduced. Meanwhile, comparisons analysis and experimental comparisons for these two different wake models are studied in sections 4 and 5. Further, the wind rose and wind Weibull probability density distribution in luxembourg are presented in section 6. Finally, the conclusion is summarized in section 7.

\section{An Improved Frandsen Wake Model(IFWM) for WFs}

An Improved Frandsen Wake Model(IFWM) is shown in Fig. 2. In the ideal state, the sideways trapezoidal region denotes the area of the far wake. The near field followed $W T_{1 i}$ (radius is $r_{1 i}$ ) can be treated as a turbulent wake. In the down-wind distance $x$, the circular cross-section radius is $r_{x i}$. The wind speed on $P_{1 i}$ and $P_{2 i}$ are assumed to be $v_{0 i}$, and the wind speed on $S_{x i}$ is $v_{x i}$.

The model assumes that in an ideal state. The distribution of wind speed is identical on every cross-section, and the far wake region spreads with a linear way. In IFWM, the tube includes the near wake region, see the green rectangular area in Fig. 2. A main fault of this tube is that the conservation of mass does not hold. The rate of mass flow of fluid entering the tube is equal to $\sum_{i=1}^{n} \rho \pi r_{x}^{2} v_{0 i}$, after go through the wind turbine, the leaving rate of mass in tube is equal to $\sum_{i=1}^{n} \rho \pi r_{x}^{2} v_{x i}$. The IFWM assumes:

$$
\begin{aligned}
\sum_{i=1}^{n} \dot{m}_{i} & =\sum_{i=1}^{n} \frac{\partial m_{i}}{\partial t_{i}} \\
& =\sum_{i=1}^{n} \rho \pi r_{x}^{2} v_{x i}, \quad n=1,2, \cdots, N
\end{aligned}
$$

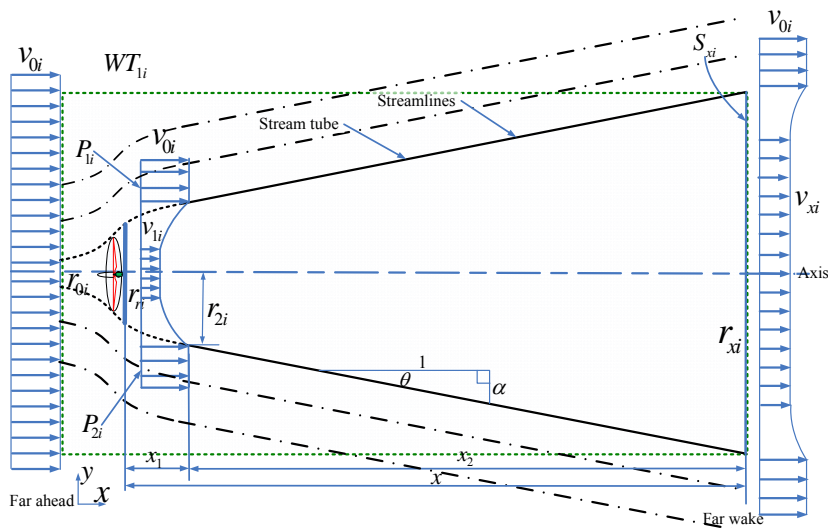

Fig. 2: The portrait of Improved Frandsen Wake Model (IFWM) with stream tube [14].

By conservation of momentum, we can obtain:

$$
\begin{aligned}
\sum_{i=1}^{n} T_{i} & =\sum_{i=1}^{n}\left(\dot{m}_{i} v_{0 i}-\dot{m}_{i} v_{x i}\right) \\
& =\sum_{i=1}^{n}\left(\frac{\partial m_{i}}{\partial t_{i}} v_{0 i}-\frac{\partial m_{i}}{\partial t_{i}} v_{x i}\right), \quad n=1,2, \cdots, N .
\end{aligned}
$$

Supposing that the radius of the $i^{\text {th }}$ actuator disk is $r_{r i}$, we obtain the area of the $i^{t h}$ actuator disk: $A_{r i}=\pi r_{r i}^{2}$. According to the definition of thrust coefficient $C_{T i}$ :

$$
\begin{aligned}
\sum_{i=1}^{n} C_{T i} & =\sum_{i=1}^{n} \frac{T_{\text {ThrustForcei }}}{T_{\text {DynamicForcei }}} \\
& =\sum_{i=1}^{n} \frac{T_{i}}{T_{\text {imax }}} \\
& =\sum_{i=1}^{n}\left[\frac{\frac{1}{2} \rho\left(v_{0 i}^{2}-v_{x i}^{2}\right) A_{r i}}{\frac{1}{2} \rho v_{0 i}^{2} A_{r i}}\right] \\
& =\sum_{i=1}^{n}\left[\frac{2 \rho v_{0 i}^{2} A_{r i} a_{i}\left(1-a_{i}\right)}{\frac{1}{2} \rho v_{0 i}^{2} A_{r i}}\right], \quad n=1,2, \cdots, N .
\end{aligned}
$$

which is

$$
\sum_{i=1}^{n} T_{i}=\sum_{i=1}^{n} C_{T i} T_{\text {imax }}, \quad n=1,2, \cdots, N .
$$

Substituting equations (1), (3) and (4) into (2), we obtain the following equation:

$$
\sum_{i=1}^{n}\left[v_{x i}^{2}-v_{0 i} v_{x i}+\frac{1}{2} C_{T i}\left(\frac{r_{r i}}{r_{x i}}\right)^{2} v_{0 i}\right]=0, \quad n=1,2, \cdots, N .
$$

Solving (5) we can easy get:

$$
\begin{aligned}
\sum_{i=1}^{n} v_{x i} & =\sum_{i=1}^{n} \frac{v_{0 i} \pm \sqrt{v_{0 i}^{2}-2 C_{T i}\left(\frac{r_{r i}}{r_{x i}}\right)^{2} v_{0 i}^{2}}}{2} \\
= & \sum_{i=1}^{n}\left[\frac{1}{2} \pm \frac{1}{2} \sqrt{\left.1-\frac{2 C_{T i}}{\left(r_{x i} / r_{r i}\right)^{2}}\right] v_{0 i}}\right. \\
= & \sum_{i=1}^{n}\left\{1-\frac{1}{2}\left[1 \mp \sqrt{1-\frac{2 C_{T i}}{\left(r_{x i} / r_{r i}\right)^{2}}}\right]\right\} v_{0 i}, \\
& \quad=1,2, \cdots, N .
\end{aligned}
$$


and taking the possible physically root of (6), which is:

$$
\begin{gathered}
\sum_{i=1}^{n} v_{x i}=\sum_{i=1}^{n}\left\{1-\frac{1}{2}\left[1-\sqrt{1-\frac{2 C_{T i}}{\left(r_{x i} / r_{r i}\right)^{2}}}\right]\right\} v_{0 i}, \\
n=1,2, \cdots, N .
\end{gathered}
$$

The above equations give the main variables and results of the IFWM.

\section{An Improved Frandsen Normal Distribution Wake Model(IFNDWM) for WFs}

An Improved Frandsen Normal Distribution Wake Model(IFNDWM) is also called Improved Frandsen Gaussian Distribution Wake Model(IFGDWM) for WFs, which is shown in Fig. 3. There are two dotted lines $A_{i}$ and $C_{i}$ selected to be the end boundaries of the IFNDWM tube. In the IFNDWM, the far wake region is confined to a dotted line tube area, whereas the farthest boundary of the far wake region in IFNDWM extends to infinity. The wind speed on $P_{1 i}$ and $P_{2 i}$ are assumed to be $v_{0 i}$, and the wind speed on $S_{x i}$ is $v_{x i}$.

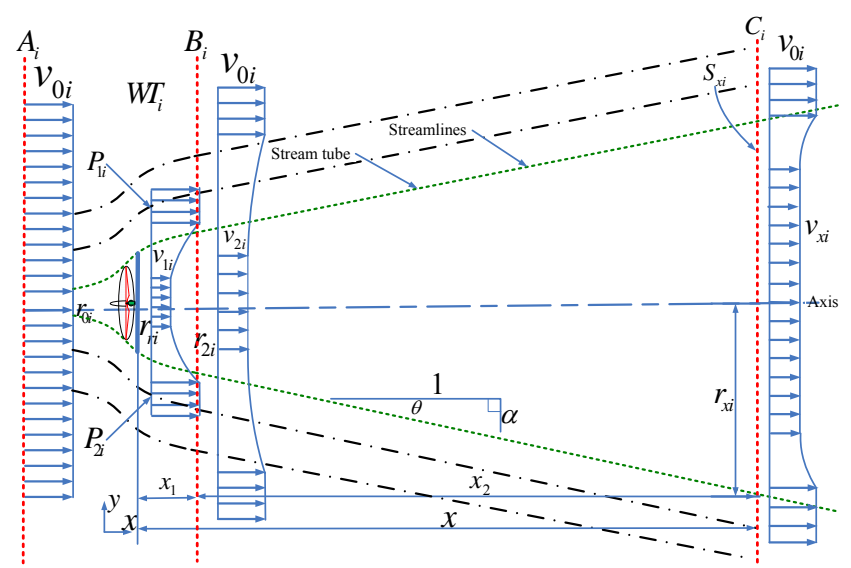

Fig. 3: The portrait of Improved Frandsen Normal Distribution Wake Model(IFNDWM) with stream.

Compare with that of IFWM, the conservation of mass is not satisfied. The rate of mass flow is assumed to be:

$$
\begin{gathered}
\sum_{i=1}^{n} \dot{m}_{i}=\frac{\partial m_{i}}{\partial t}=\sum_{i=1}^{n} \int_{0}^{+\infty} 2 \pi r_{x i} \rho v_{x_{i}, r_{x i}} d r_{x i} \\
n=1,2, \cdots, N
\end{gathered}
$$

Let us assume that IFNDWM satisfies the following equation:

$$
v_{x_{i}, r_{x i}}=\left[1-\Psi\left(x_{i}\right) \exp \left(-\frac{r_{i}^{2}}{2 \sigma_{i}^{2}}\right)\right] v_{0 i}, \quad i=1,2, \cdots, N .
$$

The parameter $\sigma_{i}$ is its standard deviation with its variance. It is also called the characteristic width in this IFNDWM. $\Psi\left(x_{i}\right)$ is a pending coefficient depending on $x_{i}$.

According to conservation of momentum, we obtain

$$
\begin{aligned}
& \sum_{i=1}^{n} \int_{0}^{+\infty} 2 \pi r_{x i} \rho v_{x_{i}, r_{x i}}\left(v_{0 i}-v_{x i, r_{x i}}\right) d r_{x i}= \sum_{\substack{i=1 \\
n}}^{n} T_{i}, \\
& n=1,2, \cdots, N .
\end{aligned}
$$

which is

$$
\begin{aligned}
\sum_{i=1}^{n} \lim _{b \rightarrow+\infty} \int_{0}^{b} 2 \pi r_{x i} \rho v_{x_{i}, r_{x i}}\left(v_{0 i}-v_{x i, r_{x i}}\right) d r_{x i}= & \sum_{i=1}^{n} T_{i}, \\
& n=1,2, \cdots, N .
\end{aligned}
$$

Substituting two equations (12) and (9) into (11),

$$
\begin{aligned}
T_{i} & =C_{T i} T_{i_{\max }} \\
& =\frac{1}{2} \rho A_{r i} v_{0 i}^{2} C_{T i} \\
& =\frac{1}{2} \rho \pi r_{r i}^{2} v_{0 i}^{2} C_{T i}, \quad n=1,2, \cdots, N .
\end{aligned}
$$

we obtain

$$
\begin{array}{r}
\sum_{i=1}^{n} \lim _{b \rightarrow+\infty} \int_{0}^{b} 2 \pi r_{x i} \rho v_{x_{i}, r_{x i}}\left(v_{0 i}-v_{x i, r_{x i}}\right) d r_{x i}= \\
\sum_{i=1}^{n} C_{T i} T_{i_{\max }}, \\
n=1,2, \cdots, N .
\end{array}
$$

Calculating this equation,

$$
\begin{aligned}
& \sum_{i=1}^{n} \lim _{b \rightarrow+\infty} \int_{0}^{b} 2 \pi r_{x i} \rho v_{0 i}^{2}\left[1-\Psi\left(x_{i}\right) \exp \left(-\frac{r_{i}^{2}}{2 \sigma_{i}^{2}}\right)\right] d r_{i} \\
& -\sum_{i=1}^{n} \lim _{b \rightarrow+\infty} \int_{0}^{b} 2 \pi r_{x i} \rho v_{0 i}^{2}\left[1-\Psi\left(x_{i}\right) \exp \left(-\frac{r_{i}^{2}}{2 \sigma_{i}^{2}}\right)\right]^{2} d r_{i} \\
& =\sum_{i=1}^{n} \frac{1}{2} \rho \pi r_{r i}^{2} v_{0 i}^{2} C_{T i}, \quad n=1,2, \cdots, N .
\end{aligned}
$$

which is

$$
\begin{aligned}
& \sum_{i=1}^{n} \lim _{b \rightarrow+\infty} \int_{0}^{b} 2 r_{x i}\left[1-\Psi\left(x_{i}\right) \exp \left(-\frac{r_{i}^{2}}{2 \sigma_{i}^{2}}\right)\right] d r_{i} \\
& -\sum_{i=1}^{n} \lim _{b \rightarrow+\infty} \int_{0}^{b} 2 r_{x i}\left[1-\Psi\left(x_{i}\right) \exp \left(-\frac{r_{i}^{2}}{2 \sigma_{i}^{2}}\right)\right]^{2} d r_{i} \\
& =\sum_{i=1}^{n} \frac{1}{2} r_{r i}^{2} C_{T i}, \quad n=1,2, \cdots, N .
\end{aligned}
$$

Computing this equation,

$$
\begin{aligned}
& \left.\sum_{i=1}^{n} \lim _{b \rightarrow+\infty} \frac{r_{x i}}{2}\right|_{0} ^{b}-\sum_{i=1}^{n} \lim _{b \rightarrow+\infty} \int_{0}^{b} \Psi\left(x_{i}\right) \exp \left(-\frac{r_{i}^{2}}{2 \sigma_{i}^{2}}\right) r_{i} d r_{i} \\
& -\left.\sum_{i=1}^{n} \lim _{b \rightarrow+\infty} \frac{r_{x i}}{2}\right|_{0} ^{b}+2 \sum_{i=1}^{n} \lim _{b \rightarrow+\infty} \int_{0}^{b} \Psi\left(x_{i}\right) \exp \left(-\frac{r_{i}^{2}}{2 \sigma_{i}^{2}}\right) r_{i} d r_{i} \\
& -\sum_{i=1}^{n} \lim _{b \rightarrow+\infty} \int_{0}^{b} \Psi^{2}\left(x_{i}\right) \exp \left(-\frac{2 r_{i}^{2}}{2 \sigma_{i}^{2}}\right) r_{i} d r_{i} \\
& =\sum_{i=1}^{n} \frac{1}{4} r_{r i}^{2} C_{T i}, \quad n=1,2, \cdots, N .
\end{aligned}
$$

Which is,

$$
\begin{aligned}
\sum_{i=1}^{n}\left[\Psi^{2}\left(x_{i}\right)-2 \Psi\left(x_{i}\right)+\frac{1}{2} C_{T i}\left(\frac{r_{r i}}{\sigma_{i}}\right)^{2}\right]= & , \\
& n=1,2, \cdots, N .
\end{aligned}
$$


That also is

$$
\sum_{i=1}^{n}\left[\Psi^{2}\left(x_{i}\right)-2 \Psi\left(x_{i}\right)+\frac{C_{T i}}{2\left(\frac{\sigma_{i}}{r_{r i}}\right)^{2}}\right]=0, \quad n=1,2, \cdots, N .
$$

Solving equation (18), we obtain

$$
\begin{aligned}
\Psi\left(x_{i}\right) & =\frac{2 \pm \sqrt{4-2 \frac{C_{T i}}{\left(\sigma_{i} / r_{r i}\right)^{2}}}}{2} \\
& =1 \pm \sqrt{1-\frac{C_{T i}}{2\left(\sigma_{i} / r_{r i}\right)^{2}}} \\
& =1 \pm \sqrt{1-\frac{C_{T i}}{2}\left(\frac{r_{r i}}{\sigma_{i}}\right)^{2}}, \quad i=1,2, \cdots, N .
\end{aligned}
$$

Taking the possible physically root, we have

$$
\Psi\left(x_{i}\right)=1-\sqrt{1-\frac{C_{T i}}{2}\left(\frac{r_{r i}}{\sigma_{i}}\right)^{2}}, \quad i=1,2, \cdots, N .
$$

Substituting the equation (20) into (9), we obtain

$$
\begin{aligned}
& v_{x_{i}, r_{x i}}=\left\{1-\left[1-\sqrt{1-\frac{C_{T i}}{2}\left(\frac{r_{r i}}{\sigma_{i}}\right)^{2}}\right] \exp \left(-\frac{r_{i}^{2}}{2 \sigma_{i}^{2}}\right)\right\} v_{0 i}, \\
& i=1,2, \cdots, N .
\end{aligned}
$$

Here, $\sigma_{i}$ is assumed a linear function of $x_{i}$ in IFNDWM. In IFWM and IFNDWM, owing to every plane is perpendicular to the axis, the rate of mass flow is equal between IFWM and IFNDWM. According to conservation of mass, we obtain

$$
\begin{aligned}
& \sum_{i=1}^{n} \int_{0}^{+\infty} 2 \pi r_{i} \rho_{i} v_{x_{i}, r_{x i}} d r_{i} \\
& =\sum_{i=1}^{n} \int_{0}^{r_{x i}} 2 \pi r_{i} \rho_{i} v_{x i} d r_{i}+\sum_{i=1}^{n} \int_{r_{x i}}^{+\infty} 2 \pi r_{i} \rho_{i} v_{0 i} d r_{i}, \\
& \quad n=1,2, \cdots, N .
\end{aligned}
$$

Which is

$$
\begin{aligned}
& \sum_{i=1}^{n} \lim _{b \rightarrow+\infty} \int_{0}^{b} 2 \pi r_{i} \rho_{i} v_{x_{i}, r_{x i}} d r_{i} \\
& =\sum_{i=1}^{n} \int_{0}^{r_{x i}} 2 \pi r_{i} \rho_{i} v_{x i} d r_{i}+\sum_{i=1}^{n} \lim _{b \rightarrow+\infty} \int_{r_{x i}}^{b} 2 \pi r_{i} \rho_{i} v_{0 i} d r_{i}, \\
& \quad n=1,2, \cdots, N .
\end{aligned}
$$

Substituting the equation (7) and (21) into (23), we obtain

$$
\begin{aligned}
& \sum_{i=1}^{n} \lim _{b \rightarrow+\infty} \int_{0}^{b} 2 \pi r_{i} \rho_{i}\{1 \\
& \left.\quad-\left[1-\sqrt{1-\frac{C_{T i}}{2}\left(\frac{r_{r i}}{\sigma_{i}}\right)^{2}}\right] \exp \left(-\frac{r_{i}^{2}}{2 \sigma_{i}^{2}}\right)\right\} v_{0 i} d r_{i} \\
& =\sum_{i=1}^{n} \int_{0}^{r_{x i}} 2 \pi r_{i} \rho_{i}\left\{1-\frac{1}{2}\left[1-\sqrt{1-\frac{2 C_{T i}}{\left(r_{x i} / r_{r i}\right)^{2}}}\right]\right\} v_{0 i} d r_{i} \\
& \quad+\sum_{i=1}^{n} \lim _{b \rightarrow+\infty} \int_{r_{x i}}^{b} 2 \pi r_{i} \rho_{i} v_{0 i} d r_{i}, \quad n=1,2, \cdots, N .
\end{aligned}
$$

Simplify the equation,

$$
\begin{aligned}
& \sum_{i=1}^{n} \lim _{b \rightarrow+\infty} \int_{0}^{b} r_{i}\{1 \\
& \quad-\left[1-\sqrt{\left.\left.1-\frac{C_{T i}}{2}\left(\frac{r_{r i}}{\sigma_{i}}\right)^{2}\right] \exp \left(-\frac{r_{i}^{2}}{2 \sigma_{i}^{2}}\right)\right\} d r_{i}}\right. \\
& =\sum_{i=1}^{n} \int_{0}^{r_{x i}} r_{i}\left\{1-\frac{1}{2}\left[1-\sqrt{1-\frac{2 C_{T i}}{\left(r_{x i} / r_{r i}\right)^{2}}}\right]\right\} d r_{i} \\
& \quad+\sum_{i=1}^{n} \lim _{b \rightarrow+\infty} \int_{r_{x i}}^{b} r_{i} d r_{i}, \quad n=1,2, \cdots, N .
\end{aligned}
$$

Which is

$$
\begin{aligned}
& \sum_{i=1}^{n}\left[1-\sqrt{\left.1-\frac{C_{T i}}{2}\left(\frac{r_{r i}}{\sigma_{i}}\right)^{2}\right] \sigma_{i}^{2}}\right. \\
& =\sum_{i=1}^{n} \frac{r_{x i}^{2}}{4}-\sum_{i=1}^{n} \frac{r_{x i}^{2}}{4} \sqrt{1-\frac{2 C_{T i}}{\left(r_{x i} / r_{r i}\right)^{2}}} \\
& \quad n=1,2, \cdots, N .
\end{aligned}
$$

Continue calculating and rearranging the equation,

$$
\begin{aligned}
& \sum_{i=1}^{n}\left[\sigma_{i}^{2}-\sigma_{i} \sqrt{\sigma_{i}^{2}-\frac{C_{T i} r_{r i}^{2}}{2}}\right] \\
& =\sum_{i=1}^{n} \frac{r_{x i}^{2}}{4}-\sum_{i=1}^{n} \frac{r_{x i}^{2}}{4} \sqrt{1-\frac{2 C_{T i}}{\left(r_{x i} / r_{r i}\right)^{2}}} \\
& \quad n=1,2, \cdots, N .
\end{aligned}
$$

Continue calculating and simplify the equation,

$$
\begin{aligned}
& \sum_{i=1}^{n} \sigma_{i}^{2}=\sum_{i=1}^{n}\left[\frac{-\frac{r_{x i}^{4}}{16}\left(1-\sqrt{1-\frac{2 C_{T i}}{\left(r_{x i} / r_{r i}\right)^{2}}}\right)^{2}}{-\frac{r_{x i}^{2}}{2}\left(1-\sqrt{1-\frac{2 C_{T i}}{\left(r_{x i} / r_{r i}\right)^{2}}}\right)+\frac{C_{T i} r_{r i}^{2}}{2}}\right] \\
& n=1,2, \cdots, N .
\end{aligned}
$$

Which is

$$
\begin{aligned}
& \sum_{i=1}^{n} \sigma_{i}^{2}=\sum_{i=1}^{n}\left[\frac{\frac{r_{x i}^{2}}{4}\left(-\frac{r_{x i}^{2}}{2}+\frac{r_{x i}^{2}}{2} \sqrt{1-\frac{2 C_{T i}}{\left(r_{x i} / r_{r i}\right)^{2}}}+\frac{C_{T i} r_{r i}^{2}}{2}\right)}{-\frac{r_{x i}^{2}}{2}+\frac{r_{x i}^{2}}{2} \sqrt{1-\frac{2 C_{T i}}{\left(r_{x i} / r_{r i}\right)^{2}}}+\frac{C_{T i} r_{r i}^{2}}{2}}\right] \\
& n=1,2, \cdots, N .
\end{aligned}
$$

Keep calculating and simplifying the equation, we obtain

$$
\sigma_{i}^{2}=\frac{r_{x i}^{2}}{4}, \quad i=1,2, \cdots, N
$$

Taking the possible physically root, we have

$$
\begin{aligned}
\sigma_{i} & =\frac{r_{x i}}{2} \\
& =\frac{r_{0 i}+\alpha_{i} x_{i}}{2} \\
& =\frac{r_{0 i}}{2}+\frac{\alpha_{i}}{2} x_{i}, \quad i=1,2, \cdots, N .
\end{aligned}
$$

where $r_{x i}=r_{0 i}+\alpha_{i} x_{i}, \alpha_{i}$ and $r_{0 i}$ can be given and estimated empirically from $\alpha_{i}=\frac{0.5}{\ln \left(z_{h i} / z_{0 i}\right)}$ and $r_{0 i}=\frac{4}{5} r_{2 i}$ in [15], respectively. Finally, equations (9), (20), (21) and (31) constitute the IFNDWM. 


\section{Comparisons of Different Wake Models for WFs}

In this section, we will discuss the relationship between IFWM and IFNDWM. Usually, comparing the Wind Speed Deficit(WSD) is a very convenient method in different of Wake Models. The WSD of WFs is defined by the following equation:

$$
\frac{\Delta v_{i}}{v_{0 i}}=\frac{v_{0 i}-v_{x i}}{v_{0 i}}, \quad i=1,2, \cdots, N
$$

Firstly, the WSD of IFWM is calculated by the equations (7) and (32):

$$
\begin{aligned}
\Delta v_{I F W M_{i}} & =\frac{v_{0 i}-v_{x_{i}}}{v_{0 i}} \\
& =\frac{v_{0 i}-\left\{1-\frac{1}{2}\left[1-\sqrt{1-\frac{2 C_{T i}}{\left(r_{x i} / r_{r i}\right)^{2}}}\right]\right\} v_{0 i}}{v_{0 i}} \\
& =\frac{1}{2}\left[1-\sqrt{1-\frac{2 C_{T i}}{\left(r_{x i} / r_{r i}\right)^{2}}}\right], \quad i=1,2, \cdots, N .
\end{aligned}
$$

After that, we calculate the WSD of IFNDWM. On the axis, with the condition: $r_{i}=0$, the WSD of IFNDWM is calculated based on the equations (21) and (32):

$$
\begin{aligned}
& \Delta v_{I F N D W M_{i}}=\frac{v_{0 i}-v_{x_{i}, r_{i}}}{v_{0 i}} \\
& =\frac{v_{0 i}-\left\{1-\left[1-\sqrt{\left.\left.1-\frac{C_{T i}}{2}\left(\frac{r_{r i}}{\sigma_{i}}\right)^{2}\right] \exp \left(-\frac{r_{i}^{2}}{2 \sigma_{i}^{2}}\right)\right\} v_{0 i}}\right.\right.}{v_{0 i}} \\
& =1-\sqrt{1-\frac{C_{T i}}{2}\left(\frac{r_{r i}}{\sigma_{i}}\right)^{2}} \\
& =1-\sqrt{1-\frac{C_{T i}}{2\left(\frac{\sigma_{i}}{r_{r i}}\right)^{2}}, \quad i=1,2, \cdots, N .}
\end{aligned}
$$

Substituting $\sigma_{i}=\frac{r_{x i}}{2}$ into (34), we obtain

$$
\begin{aligned}
\Delta v_{I F N D W M_{i}} & =\frac{v_{0 i}-v_{x_{i}, r_{i}}}{v_{0 i}} \\
& =1-\sqrt{1-\frac{C_{T i}}{2}\left(\frac{r_{r i}}{\left.\frac{r_{x i}}{2}\right)^{2}}\right.} \\
& =1-\sqrt{1-\frac{2 C_{T i}}{\left(r_{x i} / r_{r i}\right)^{2}}}, \quad i=1,2, \cdots, N .
\end{aligned}
$$

According to the equations (33) and (35), the ratio of the WSD from IFWM to IFNDWM is calculated by

$$
\begin{aligned}
\frac{\Delta v_{I F W M_{i}}}{\Delta v_{I F N D W M_{i}}} & =\frac{\frac{1}{2}\left[1-\sqrt{1-\frac{2 C_{T i}}{\left(r_{x i} / r_{r i}\right)^{2}}}\right]}{1-\sqrt{1-\frac{2 C_{T i}}{\left(r_{x i} / r_{r i}\right)^{2}}}} \\
& =\frac{1}{2}, \quad i=1,2, \cdots, N .
\end{aligned}
$$

We can see that on the axis, the WSD of IFWM is half as small as that of IFNDWM.

\section{Experimental Comparisons of Different Wake Models for WFs}

In this section, we analyze five cases to validate IFWM and IFNDWM. In the literature [16], the Large-Eddy Simulation (LES) data and a miniature WT were used as the standard case. The main parameters of the five cases are shown in the following table (1).

Table 1: Different experiments and LES case to validate the improved model of WFs in Luxembourg [16, 17].

\begin{tabular}{llllllll}
\hline Cases & $d_{r i}(\mathrm{~m})$ & $z_{h i}(\mathrm{~m})$ & $v_{0 i}(\mathrm{~m} / \mathrm{s})$ & $C_{T i}$ & $z_{0 i}(\mathrm{~m})$ & $\alpha_{i}$ & $r_{0 i}(\mathrm{~m})$ \\
\hline \hline Case $_{a}$ & 0.15 & 0.125 & 2.2 & 0.42 & 0.00003 & 0.046 & 0.066 \\
Case $_{b}$ & 66 & 70 & 9 & 0.8 & 0.5 & 0.11 & 38.08 \\
Case $_{c}$ & 66 & 98 & 9 & 0.8 & 0.05 & 0.08 & 40.48 \\
Case $_{d}$ & 82 & 70 & 9 & 0.8 & 0.005 & 0.06 & 43.52 \\
Case $_{e}$ & 70 & 70 & 9 & 0.8 & 0.00005 & 0.062 & 41.12 \\
\hline
\end{tabular}

Note: Case $_{b}$ represents WF Kehmen-Heischent; Case $_{c}$ represents WF Hengischt S.A; Case $_{d}$ represents WF Binsfeld; Case $_{e}$ represents WF Burer Bierg.

Using some data in table (1), we have done the simulation experiments. Analysing the Figs. 4(a) and 4(b), we can find that WSD of IFNDWM are twice as larger as that of IFWM. Going through these simulations, which have provided to test and verify the WSD of IFWM are half as small as that of IFNDWM on the axis. These simulations are in good agreement with the correctness of the models in last sections.

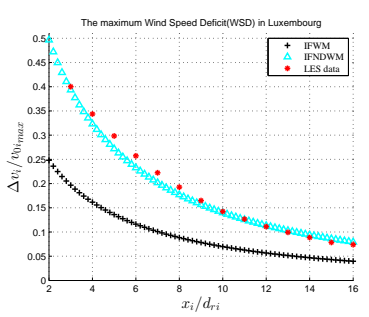

(a) Maximum WSD in Case $_{a}$

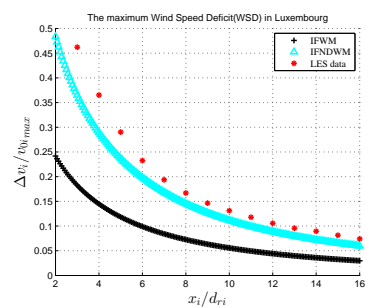

(b) Maximum WSD in Case $_{c}$ Fig. 4: The portrait of the maximum Wind Speed Deficit(WSD) of Case ${ }_{a}$ and Case $_{c}$ in Luxembourg.

\section{Wind Rose and Wind Weibull Probability Den- sity Distribution}

In this section, we describe and summarize the wind rose and wind Weibull probability density distribution focusing on WFs of Luxembourg. We get the actual and real-time data from this Website [18]. Some WE cases are reduced and the WR is simplified through merging the wind speeds and wind directions in Luxembourg. These data of WE resource are collected and sorted out in the following figures. The Fig. 5(a) shows rose portraits of average WD and WD (North=0) in Luxembourg from Jan. 01 to Dec. 31, 2015. The probabilities of most of WDs from southwest are more than $4 \%$ and approximate approaches to 5\%. Whereas, a small amount of WD from northeast are more than $4 \%$ and approximate approaches to $5 \%$. Therefore, WTs should face to southwest in WFs of Luxembourg in summer time, another situation is just the opposite in winter. 
The wind rose shows the portraits of wind speed $(\mathrm{m} / \mathrm{s})$ and wind direction in Luxembourg from Jan. 01 to Dec. 31, 2015 in Fig. 5(a). The most of wind mean speeds from southwest are more than $6 \mathrm{~m} / \mathrm{s}$. Whereas, a small amount of wind mean speed from northwest are approximate to $6 \mathrm{~m} / \mathrm{s}$. Therefore, wind turbines should face to southwest in WFs of Luxembourg.

We continue getting the actual and real-time data from this Website [18]. The wind speed in Luxembourg are collected from Jan. 01 to Dec. 31 in 2015 and their Mean Wind Speed (MWS) is $3.5410 m / s$. They are shown in the Fig. 5(b).
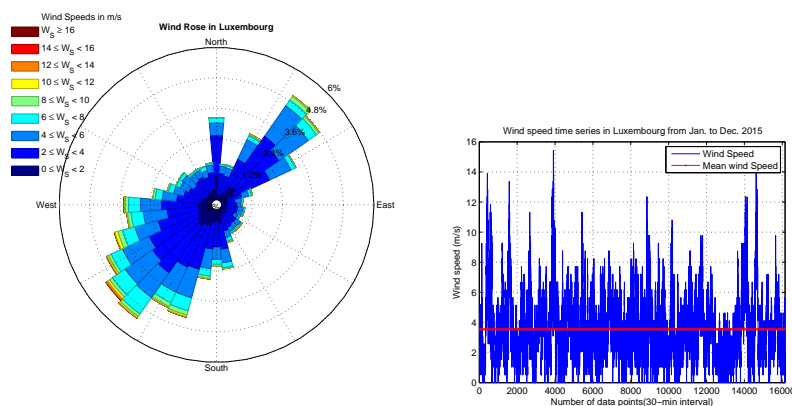

(a) $\mathrm{WD}(\mathrm{North}=0)$ and $\operatorname{speed}(\mathrm{m} / \mathrm{s})$

(b) Wind speed time series and mean speed

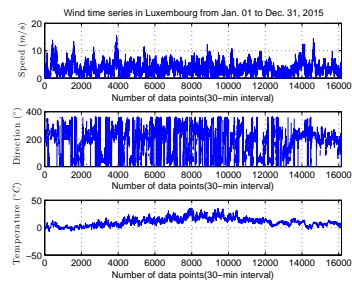

(c) Wind situations time series future.

\section{References}

[1] J. R. Marden, S. D. Ruben, and L. Y. Pao, "A model-free approach to wind farm control using game theoretic methods," IEEE Transactions on Control Systems Technology, vol. 21, no. 4, pp. 1207-1214, 2013.

[2] R. Shakoor, M. Y. Hassan, A. Raheem, and Y.-K. Wu, "Wake effect modeling: A review of wind farm layout optimization using Jensen's model," Renewable and Sustainable Energy Reviews, vol. 58, pp. 1048-1059, 2016.

[3] S. Kuenzel, L. Kunjumuhammed, B. Pal, and I. Erlich, "Impact of wakes on wind farm inertial response," Sustainable Energy, IEEE Transactions on, vol. 5, no. 1, pp. 237-245, Jan 2014.

[4] L. Y. Pao and K. Johnson, "A tutorial on the dynamics and control of wind turbines and wind farms," in American Control Conference, 2009. ACC'09., June 2009, pp. 2076-2089.

[5] E. Bitar and P. Seiler, "Coordinated control of a wind turbine array for power maximization," in American Control Conference (ACC), 2013, June 2013, pp. 2898-2904.

[6] L. Pan, H. Voos, Y. Li, M. Darouach, Y. Xu, and S. Hu, "A wake interaction model for the coordinated control of wind farms," in Emerging Technologies Factory Automation (ETFA), 2015 IEEE 20th Conference on, Sept 2015, pp. 1-7.

[7] L. Pan, H. Voos, Y. Li, Y. Xu, M. Darouach, and Z. Li, "A class of improved wake interaction model for the coordinated control of wind farms," in Chinese Automation Congress (CAC), 2015, Nov 2015, pp. 1322-1327.

[8] J. Park and K. H. Law, "A data-driven, cooperative wind far$\mathrm{m}$ control to maximize the total power production," Applied Energy, vol. 165, pp. 151-165, 2016.

[9] G. R. Marseglia, A. Arbasini, S. Grassi, M. Raubal, and D. M. Raimondo, "Optimal placement of wind turbines on a continuous domain: An milp-based approach," in American Control Conference (ACC), 2015, July 2015, pp. 5010-5015.

[10] ENOVOS, "Enovos," http://www.enovos.eu.

[11] T. Wind Power, "The Wind Power," http://www.thewindpower.net/index.php.

[12] A. M. Howlader, T. Senjyu, and A. Y. Saber, "An integrated power smoothing control for a grid-interactive wind farm considering wake effects," IEEE Systems Journal, vol. 9, no. 3, pp. 954-965, Sept 2015.

[13] H. Gu, J. Wang, Q. Lin, and Q. Gong, "Automatic contourbased road network design for optimized wind farm micrositing," IEEE Transactions on Sustainable Energy, vol. 6, no. 1, pp. 281-289, Jan 2015.

[14] S. Frandsen, R. Barthelmie, S. Pryor, O. Rathmann, S. Larsen, J. Højstrup, and M. Thøgersen, "Analytical modelling of wind speed deficit in large offshore wind farms," Wind energy, vol. 9, no. 1-2, pp. 39-53, 2006.

[15] S. Pookpunt and W. Ongsakul, "Optimal placement of wind turbines within wind farm using binary particle swarm optimization with time-varying acceleration coefficients," Renewable Energy, vol. 55, pp. 266-276, 2013.

[16] M. Bastankhah and F. Port-Agel, "A new analytical model for wind-turbine wakes," Renewable Energy, vol. 70, pp. 116123, 2014.

[17] L. Sizhuang and F. Youtong, "Analysis of the Jensen's model, the Frandsen's model and their Gaussian variations," in Electrical Machines and Systems (ICEMS), 2014 17th International Conference on, Oct 2014, pp. 3213-3219.

[18] I. S. U. of Science and Technology, "The Iowa Environmental Mesonet (IEM)," http://mesonet.agron.iastate.edu/. 